\title{
The MROI fringe tracker: laboratory tracking with ICONN
}

\author{
T. M. McCracken ${ }^{a}$, C. A. Jurgenson ${ }^{a}$, J. S. Young ${ }^{c}$, E. B. Seneta ${ }^{c}$, D. F. Buscher ${ }^{c}$, C. A. \\ Haniff $^{c}$, M. J. Creech-Eakman ${ }^{b}$, F. G. Santoro ${ }^{d}$, A. V. Shtromberg ${ }^{b}$, L. M. Schmidt ${ }^{b}$, S. \\ Rochelle ${ }^{b}$ \\ ${ }^{a}$ Yale University, Astronomy Department, 260 Whitney Avenue, New Haven, CT 06511, USA; \\ ${ }^{b}$ New Mexico Institute of Mining and Technology, Magdalena Ridge Observatory, 801 Leroy \\ Place, Socorro, NM 87801, USA; \\ ${ }^{c}$ University of Cambridge, Cavendish Laboratory, Dept. of Physics, JJ Thomson Avenue, \\ Cambridge, CB3 OHE, UK; \\ ${ }^{d}$ Giant Magellan Telescope Project, 251 S. Lake Avenue, Suite 300, Pasadena, California \\ 91101, USA
}

\begin{abstract}
The loop is closed on ICONN, the Magdalena Ridge Observatory Interferometer fringe tracker. Results from laboratory experiments demonstrating ICONN's ability to track realistic, atmospheric-like path difference perturbations in real-time are shown. Characterizing and understanding the behavior and limits of ICONN in a controlled environment are key for reaching the goals of the MROI. The limiting factors in the experiments were found to be the light delivery system and temporary path length correction mechanism; not the on-sky components of ICONN. ICONN was capable of tracking fringes with a coherence loss below $5 \%$; this will only improve in its final deployment.
\end{abstract}

Keywords: MROI, optical interferometry, fringe tracker, fringe tracking

\section{INTRODUCTION}

The heart of the Magdalena Ridge Observatory Interferometer ${ }^{1}$ (MROI, see paper 9146-17 of these proceedings) is its fringe tracker, the Infrared Coherencing Nearest Neighbor tracker (ICONN). ${ }^{2}$ ICONN is a dedicated fringe tracking beam combiner operating in the $H$ and $K_{\mathrm{s}}$ bandpasses. It is based on the pairwise, nearest neighbor, bootstrapping scheme ${ }^{3}$ which phases the array on the shortest baselines. This scheme maximizes the fringe visibility available to track on. To realize MROI's science goals and push its limiting magnitude beyond the current state-of-the-art, ICONN's performance must be maximized.

Ground based amplitude interferometers need fringe trackers to correct the random path length variations introduced by the atmosphere. These aberrations limit how faint a source an interferometer can detect. Pushing arrays to fainter magnitudes means optimizing their fringe trackers. For ICONN, this process starts in the lab. We have setup an experiment that simulates telescope inputs, the delay lines, and the beam combination of MROI, enabling us to close the loop on ICONN and track fringes in a controlled laboratory environment. This allows us to identify software bottlenecks and optimize ICONNS's tracking algorithms prior to observatory deployment. It also allows us to better define and test its interfaces with the overall MROI supervisory control software $^{4}$ and other MROI sub-systems e.g. the Automated Alignment System (AAS). ${ }^{5,6}$

We present here a review of the laboratory experiment in which the the loop was closed on ICONN and its initial results. Due to extended vendor delays, a portion of the ICONN beam train had to be temporarily modified in order to close the loop. The effect these modifications had on the data product are explored in the context of the experiment and ICONN's performance is extracted from the data. We are continuing to optimize and characterize ICONN, all with the goal of tracking fringes with less than a $1 \%$ visibility loss and push MROI as a whole to its limit.

Further author information:

T.M.M: E-mail: tyler.mccracken@yale.edu

Optical and Infrared Interferometry IV, edited by Jayadev K. Rajagopal, Michelle J. Creech-Eakman,

Fabien Malbet, Proc. of SPIE Vol. 9146, 91461E • @ 2014 SPIE • CCC code: 0277-786X/14/\$18

doi: $10.1117 / 12.2055603$ Proc. of SPIE Vol. 9146

Proc. of SPIE Vol. 9146 91461E-1 


\section{EXPERIMENTAL CONFIGURATION}

A conceptual overview of the closed-loop fringe experiment $(\mathrm{CLFE})^{7,8}$ is given in figure 1 ; hardware subcomponents are shown as rectangles and software sub-components as elliptical objects. Only a high-level view of the software is given, with each ellipse representing the top-level component running on a particular computer. Individual paths from simulated unit telescopes are shown as solid lines. Software communication paths are either dashed lines, corresponding to time-critical communication, or dot-dashed lines, corresponding to nontime-critical software communication. Each path is labeled with its primary function. The AAS, a separate sub-system of MROI, is shown as a diamond shape.

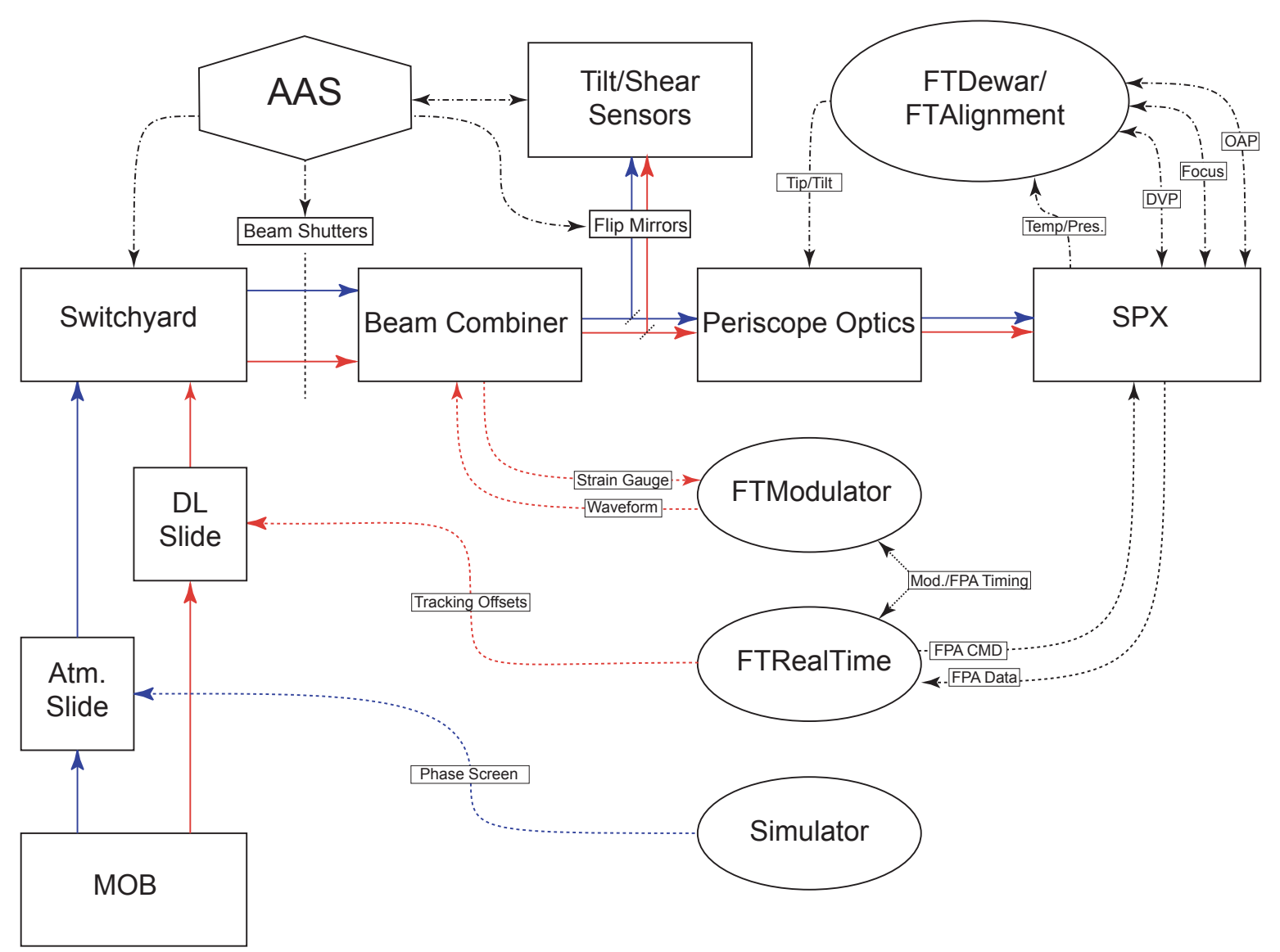

Figure 1: Conceptual drawing of the closed-loop fringe experiment. Rectangular components represent hardware sub-systems and elliptical components represent software processes. Light paths are drawn with solid lines, time-critical software flow with dashed lines, and non-time-critical software flow with the dot-dashed lines.

Figure 1 shows two distinct flows: that of the primary optical path and the time-critical data between software components used to close the loop.

The primary optical flow begins with light generated from the Magic Optical Box (MOB, a sub-component of the AAS) and transported to ICONN using a $2 \times 2$ fiber coupler. One input contains white light and the other $1550 \mathrm{~nm}$ laser light. Both outputs are collimated using Thorlabs reflective collimators mounted to either the atmospheric simulator slide or the mock delay line slide. The atmospheric slide introduces a simulated piston error. ICONN estimates the piston error from the fringe data and commands the delay line slide to eliminate the error. The switchyard aligns the beams to the beam combiner. Within the combiner, the outer path is phase modulated and combined with its inner combination partner. The periscope optics align the optical axis of the beam combiner output to that of the input optical axis of the spectrograph (SPX in the figure). The 
spectrograph disperses the combined light and focuses it onto a column of pixels on the detector, a Teledyne PICNIC array.

The time-critical software flow starts by reading the detector and transferring the pixel data to the real-time process FTRealTime. This process runs under the real-time Linux framework Xenomai and represents the brain of the fringe tracker. An Astronomical Research Cameras (ARC) controller commands detector reads. The ARC controller is synchronized to the phase modulation using a series of clocks. From the fringe data, FTRealTime calculates the optical path difference between the combination partners and commands the delay line slide to move in accordance with the delay estimate, thereby closing the loop on ICONN.

Other software communication paths shown in figure 1 include the alignment of the periscope optics to the spectrograph, selection of the observing mode, focusing/alignment of the detector, and temperature/pressure monitoring; all performed with either the FTDewar or FTAlignment process. The tilt/shear sensors, beam shutters, and flip mirrors are sub-components of the AAS and are used for alignment purposes. The complete CLFE setup is shown in figure 2 .

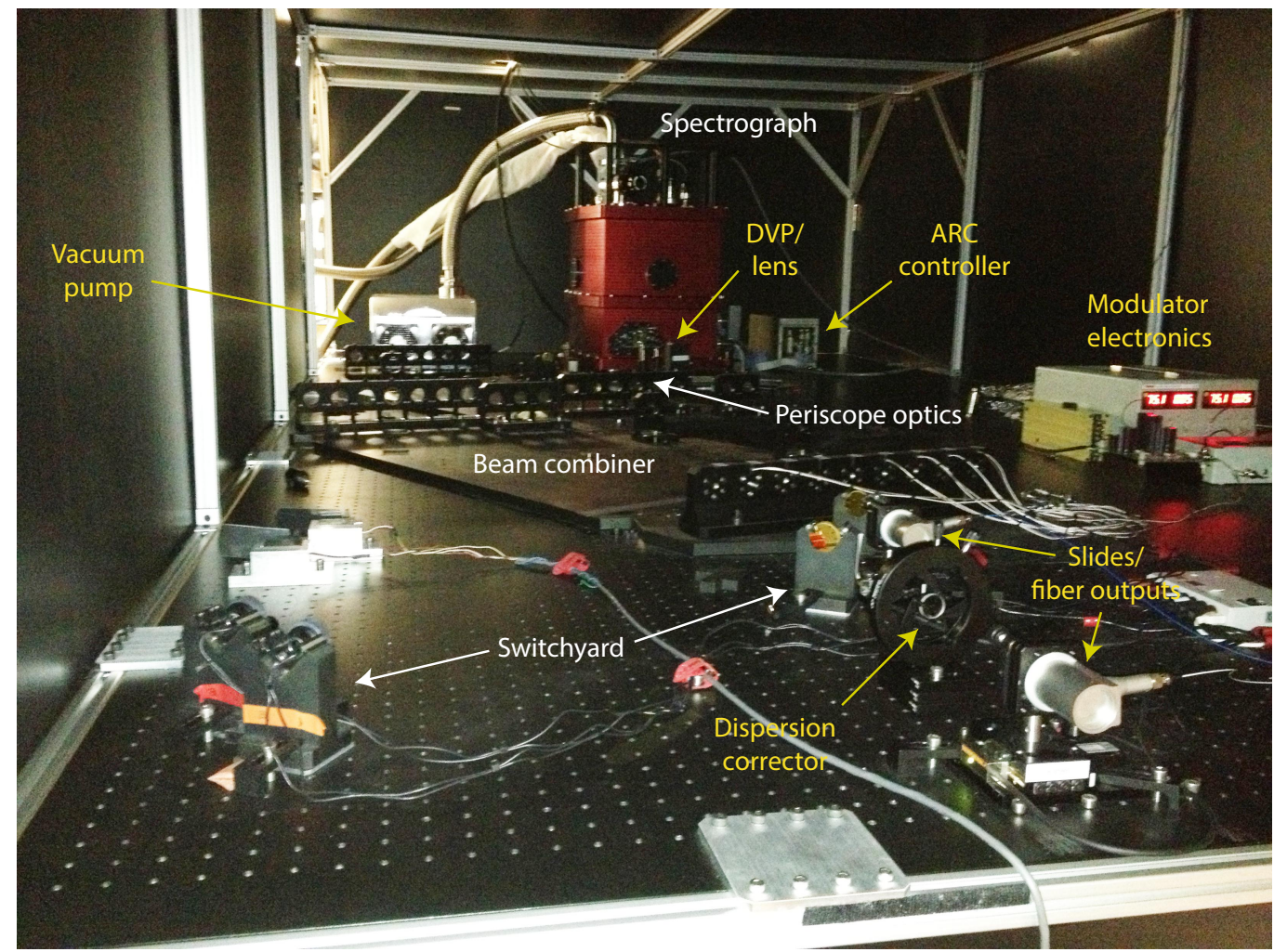

Figure 2: Laboratory setup of the CLFE.

\section{CLFE CHARACTERIZATION}

Prior to closing the loop on ICONN, there were a number of sub-components that we wanted to characterize in order to be able to realistically infer ICONN's intrinsic performance. This included characterizing temporary modifications to the ICONN beam train due to continuing vendor delays. The temporary modifications are not permanent and will be removed once the necessary components are delivered. The fiber coupler and motion slides described below are not part of ICONN so their performance does not ultimately affect that of ICONN's. They are only used in the context of the CLFE.

\subsection{Temporary modifications}

Due to extended vendor delays, the cold optics and periscope optics were not used in the initial phase of the CLFE. A temporary solution was mocked up by moving the cold optics (dispersing elements and focusing optics) 
outside of the spectrograph so we could progress and close the loop. This had a number of effects:

- To minimize thermal noise, it was decided to use only the $H$ band for fringe tracking until the cold optics were delivered. A $H$ band filter was temporarily mounted directly in front of the detector to limit the thermal background signal.

- The detector was temporarily relocated to a position near the front of the cryostat because the relay optics were not available.

- The detector would therefore be operated warmer than the nominal $80 \mathrm{~K}$.

- An off-the-shelf $\mathrm{CaF}_{2}$ lens was used for focusing light on the detector.

- The focal length of the $\mathrm{CaF}_{2}$ lens was necessarily than that of the reflective focal length of the focusing OAP in the final design. This was due to the detector being relocated.

- The bandpass of interest was spread over more pixels than the nominal five because of the modifications to the focusing optics.

- No spatial filtering was implemented.

The AAS is responsible for detecting and correcting any residual misalignment within the beam combiner by using the tilt/shear sensors and algorithms. These were not implemented. Alignment was done in the pupil plane by overlapping the beams and eliminating the tilt fringes. Regardless, some residual tip/tilt in the combined beams was expected.

\subsection{Fiber coupler}

The MOB fiber coupler presented two issues: splitting ratio and fiber length mismatch.

The manufacturer specifications on the fiber lengths was $\pm 5 \mathrm{~cm}$. This much differential path length in a dispersive medium results in significant dispersion that will wash out the fringes within a bandpass, or significantly change the position of zero group delay across a set of spectral channels. In order to assess this, various thicknesses of fused silica was introduced into a beam path and the position of the peak visibility in each spectral channel was found. A $4 \mathrm{~mm}$ piece of fused silica was found to give the best correction for the dispersion.

It was also known that the splitting ratio of the fibers, specified as 50/50 at $1550 \mathrm{~nm}$, varied away from the specified wavelength. ${ }^{5}$ An intensity mismatch between the combined beams reduces the visibility. ${ }^{9}$ To assess this, we obtained spectra and hence relative intensities of the beams. Table 1 summarizes the wavelength-dependent fiber splitting ratio with $\mathrm{S} 1$ and $\mathrm{S} 2$ representing the individual beams, $\rho_{\lambda}$ the ratio of $\mathrm{S} 1$ to $\mathrm{S} 2$, and $V_{\rho}$ is visibility reduction factor.

\begin{tabular}{|c||c|c|c|c|c|c|c|}
\hline$\lambda_{0}(\mathrm{~nm})$ & 1770 & 1734 & 1699 & 1666 & 1635 & 1587 & 1558 \\
\hline S1 (ADUs) & 119 & 319 & 627 & 1054 & 1601 & 2030 & 1723 \\
S2 (ADUs) & 2316 & 4118 & 4529 & 4316 & 3801 & 3034 & 1882 \\
$\rho_{\lambda}$ & 0.05 & 0.08 & 0.14 & 0.24 & 0.42 & 0.67 & 0.92 \\
$V_{\rho}$ & 0.43 & 0.52 & 0.65 & 0.79 & 0.91 & 0.98 & 1.00 \\
\hline
\end{tabular}

Table 1: Table detailing the splitting ratio of the fiber coupler and the effect it has on the measured visibilities.

\subsection{Motion slides}

A pair of Nanomotion slides were used for simulating the atmospheric piston error and to act as a mock delay line. The slides had an advertised encoder resolution of $100 \mathrm{~nm}$ but errors in their response were apparent. This was most notable during a fringe scan when one of the slides was scanned at constant velocity through the fringe packet with detector reads taken at regular intervals. The scanning slide had a tendency to run-off before its internal servo would correct it. This is shown on the left-hand side of figure 3. 
The right-hand side of figure 3 shows the discretization error of the slide drifting between encoder counts while held at stationary points. This behavior was expected but with an encoder count corresponding to $100 \mathrm{~nm}$, a few counts means a drift of up to $\lambda / 4$. In this particular example, the slide was driven with a square wave and the bottom portion of the waveform exhibits more discretization error.
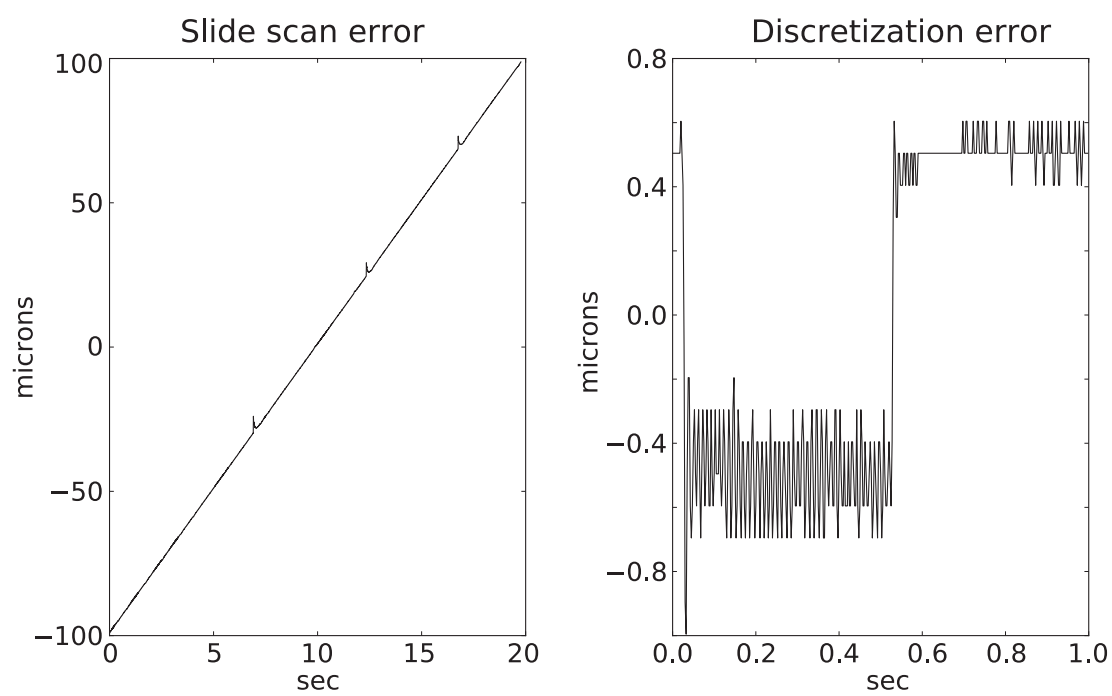

Figure 3: Encoder data from a slide scan show occasional small non-linearity. The discretization error is another limiting factor in tracking performance.

\subsection{Visibility factors}

Due to the temporary modifications and performance of the fiber coupler, we knew there would be a number of visibility reduction factors within the context of the CLFE. Sources of the visibility reductions are attributable to intensity mismatch, polarization effects, group delay error, and wavefront errors. Each of these factors were quantified and the remaining visibility reduction was attributed to alignment. This meant the CLFE visibility $\left(V_{\mathrm{CLFE}}\right)$ could be written as:

$$
V_{\mathrm{CLFE}}=V_{\rho} V_{\mathrm{pol}} V_{\mathrm{GD}} V_{\mathrm{wfe}} V_{\mathrm{align}} .
$$

\subsubsection{Polarization and group delay}

The visibility reduction due to polarization $\left(V_{\text {pol }}\right)$ for the ICONN beam combiner optics had been calculated previously. ${ }^{10}$ This factor was found to be close to unity (> 0.999). No measurements were performed to characterize the polarization effects from the switchyard, recollimating OAPs, or fiber coupler. Because the fibers are polarization maintaining, and each beam goes through an identical set of reflections from the outputs of the fibers into the beam combiner, this reduction factor was assumed to be negligible.

The group delay reduction factor $\left(V_{\mathrm{GD}}\right)$, was calculated from the dispersion measurements used to identify the correct thickness of glass to place within one of the beam paths. Using a coherence length of $50 \mu \mathrm{m}$ and a conservative estimate in the wavelength dependent change in group delay of $1 \mathrm{~nm}$ per micron (zero group delay position changes by $1 \mathrm{~nm}$ per micron of bandpass) $V_{\mathrm{GD}} \approx 0.996$.

\subsubsection{Wavefront errors}

The wavefront errors in the CLFE are summarized in table 2. The table includes the source of the error, the number of elements in the CLFE introducing that error $(N)$, the RMS wavefront error from the source, and the visibility reduction factor associated with the error from a single source at a wavelength of $1635 \mathrm{~nm}$. The reduction factor for wavefront errors through the beam combiner optics was found previously. ${ }^{10}$ The recollimating OAP had a diamond turned reflective surface; an estimate of $15 \mathrm{~nm}$ was used for this optic based on experience with diamond turning vendors. The switchyard mirrors are identical to the beam combiner mirrors, which 
have a quoted RMS error of $5 \mathrm{~nm} .^{10}$ The equivalent wavefront error, when all the contributions were added in quadrature, was found to be $49.4 \mathrm{~nm}$ resulting in a visibility factor of $V_{\text {wfe }}=0.965$ at $1635 \mathrm{~nm} .{ }^{7}$

\begin{tabular}{|r|c|c|l|}
\hline Source & $N$ & $\delta_{\mathrm{rms}}(\mathrm{nm})$ & V at $1635 \mathrm{~nm}$ \\
\hline Recollimating OAP & 2 & 21 & 0.997 \\
Switchyard mirrors & 4 & 10 & 0.999 \\
BC S1 path & 1 & 22 & 0.996 \\
BC S2 path & 1 & 26 & 0.995 \\
\hline Total & & 49.4 & 0.965 \\
\hline
\end{tabular}

Table 2: Summary of wavefront errors in the CLFE at $1635 \mathrm{~nm}$.

\subsubsection{Alignment}

The remaining visibility factor to be considered in equation 1 is alignment. With the absence of the tilt/shear correction offered by the AAS, some residual tilt and shear was expected. No direct measurement of this error was performed. To quantify the error, the Michelson visibility, $\left(I_{\max }-I_{\min }\right) /\left(I_{\max }+I_{\min }\right)$, was estimated per spectral channel from the spectral scans. All the visibility factors discussed above were removed leaving only that due to the alignment error. This is summarized in table 3 where $V_{\text {meas }}$ is the measured visibility from a spectral scan and $V_{\text {align }}$ is the visibility alignment factor.

\begin{tabular}{|l||c|c|c|c|c|c|c|}
\hline$\lambda_{0}(\mathrm{~nm})$ & 1770 & 1734 & 1699 & 1666 & 1635 & 1587 & 1558 \\
\hline$V_{\text {meas }}$ & 0.278 & 0.306 & 0.352 & 0.403 & 0.442 & 0.471 & 0.472 \\
$V_{\rho}$ & 0.43 & 0.52 & 0.65 & 0.79 & 0.91 & 0.98 & 1.00 \\
\hline$V_{\text {pol }}$ & \multicolumn{7}{|c|}{0.999} \\
$V_{\text {GD }}$ & \multicolumn{8}{|c|}{0.996} \\
\hline$V_{\text {wfe }}$ & 0.970 & 0.968 & 0.967 & 0.966 & 0.965 & 0.962 & 0.961 \\
\hline$V_{\text {align }}$ & 0.695 & 0.634 & 0.584 & 0.550 & 0.524 & 0.520 & 0.511 \\
\hline
\end{tabular}

Table 3: Summary of the visibilities in the CLFE. $V_{\text {meas }}$ is divided by each term leaving only the alignment visibility reduction factor.

The CLFE has a $12 \mathrm{~mm}$ beam diameter. To result in a tilt error producing a visibility reduction factor of 0.511 at $\lambda=1558 \mathrm{~nm}$, the angle between the wavefronts is $\alpha=0.595 \lambda / D=0.0044^{\circ}$ or 16 arcseconds. The same angle corresponds to $V_{\text {align }}=0.699$ at $1770 \mathrm{~nm}$; versus 0.695 for the measured alignment reduction. This shows that assigning the remaining visibility reduction factor to $V_{\text {align }}$ is a consistent solution. It is expected that when the tilt/shear algorithms are implemented, $V_{\text {align }}$ will be much closer to unity.

\section{RESULTS}

A summary of the tracking results from the CLFE follows. These results were obtained with the temporary modifications detailed above and were found to be limited by the fiber coupler and correction slides. With the inclusion of the cold optics and further optimization of the detector readout, we expect these results to improve. In the end, the CLFE was, and will be, limited to components external to ICONN.

\subsection{Tracking visibilities}

We used a coherencing fringe tracking algorithm ${ }^{11}$ to close the loop initially. The fringe modulation was set to a triangle wave with less than a $2 \mu \mathrm{m}$ amplitude and a $5 \mathrm{~Hz}$ period. Frames were taken in $10 \mathrm{~ms}$ intervals with a $100 \mathrm{~ms}$ coherent integration and a $200 \mathrm{~ms}$ incoherent integration. Frame rates were limited by detector timing; an issue that is currently being addressed.

The atmospheric slide was driven with a six second period, $30 \mu \mathrm{m}$ amplitude sinusoid to simulate the low frequency perturbations expected by the atmosphere. To quantify how well ICONN tracked the sinusoid, the visibility was calculated for every coherent integration. We then compared this quantity to the maximum visibility found when the fringe packet was scanned. The tracking visibility data is shown in figure 4 . Because the fringe 


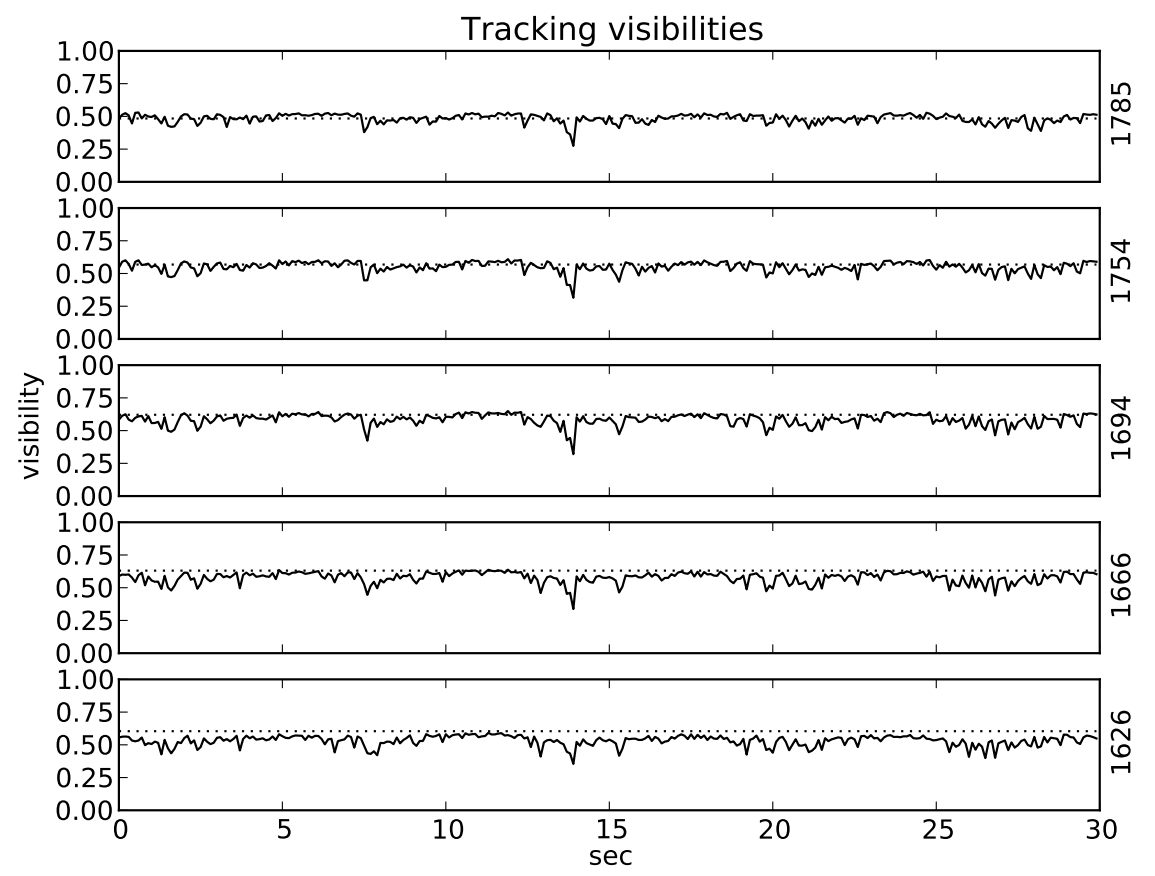

Figure 4: Estimated visibilities from the tracking experiment. The dotted line represents the visibility estimate from the fringe scan.

scan does not necessarily sample the fringe pattern at its peak, it is not an upper bound but does provide a good estimate of the maximum visibility on a per channel basis.

Figure 4 shows that over the course of 30 seconds, ICONN was not only able to track the fringe packet as it was perturbed but also maintain the contrast of the fringes. Though the estimated visibilities show some deviation from the upper bound, given the issues with the slide and the MOB fibers, ICONN was still able to perform its task at a high level.

For each spectral channel shown in figure 4, the root-mean square (RMS) and standard deviation of the measured tracking visibility is given in table 4 , along with the target visibility from the spectral scan.

\begin{tabular}{|c|c|c|c|}
\hline$\lambda$ & RMS & StD & $V_{\text {target }}$ \\
\hline 1785 & 0.486 & 0.034 & 0.484 \\
1754 & 0.553 & 0.040 & 0.568 \\
1694 & 0.587 & 0.044 & 0.621 \\
1666 & 0.579 & 0.043 & 0.630 \\
1626 & 0.533 & 0.041 & 0.604 \\
\hline
\end{tabular}

Table 4: Summary of measured visibility in the tracking experiment on a per spectral channel basis.

Apparent from table 4 is that the highest visibility is on the central spectral channel. This is expected as the algorithm tracks the point of constant phase. As the fringe packet moves back and forth, the spectral channels at the extreme ends will be the first to 'see' the packet moving because they have the largest difference in spectroscopic wavenumber from the central channel, and thus, will show a phase change quicker than spectral channels nearer the central channel. The standard deviation on the measured visibility is larger than the goal for ICONN, to track the visibility to within $1 \%$ of its value. This is elaborated on in the following. 


\subsection{ICONN performance}

The tracking visibilities showed that ICONN was performing at a high level. We wanted to expand on this and characterize ICONN's performance in the CLFE. For this, we expressed the measured visibility as a product of two visibilities, the target visibility found from the fringe scan and the tracking visibility that would tell us how well ICONN was tracking fringes:

$$
V_{\text {measured }}=V_{\text {target }} V_{\text {track }} \text {. }
$$

If ICONN and the delay line slide were to track perfectly, $V_{\text {measured }}$ would simply be $V_{\text {target }}$ as $V_{\text {track }}$ would be unity. Based on this, the tracking performance of ICONN, in conjunction with the delay line slide, may be estimated by dividing the measured visibility by that of the target visibility. This is summarized in table 5 .

\begin{tabular}{|c|c|c|}
\hline$\lambda$ & RMS & StD \\
\hline 1785 & 1.003 & 0.070 \\
1754 & 0.973 & 0.070 \\
1694 & 0.945 & 0.070 \\
1666 & 0.918 & 0.069 \\
1626 & 0.882 & 0.067 \\
\hline \hline mean & 0.944 & 0.069 \\
\hline
\end{tabular}

Table 5: Tracking visibilities per spectral channel.

When $V_{\text {track }}$ is viewed across the bandpass, it shows the longer wavelength spectral channels have a smaller contribution to the loss in tracking visibility. This is expected because the same delay was introduced in all spectral channels, resulting in the shorter wavelengths having a more pronounced phase shift.

It is insightful to view the tracking visibility factor alongside the integrated offset produced by the fringe engine. This is shown in figure 5, with the mean tracking visibility taken from all spectral channels. It is apparent that $V_{\text {track }}$ has a periodic nature to it, with the same frequency as the sinusoid but with a $\pi$ phase shift introduced.

The cause of the periodicity in $V_{\text {track }}$ is due to the errors associated with the motion of the mock delay line slide. Recall figure 3 showing the discretization error of the slide and that one side has a larger error than the other. The slide has a more difficult time in reversing directions from forwards to backwards than vice versa. When tracking, the slide causes a larger error when accelerating from and decelerating towards the local maxima on the integrated offset. This is further evident by the increased spread on $V_{\text {track }}$ at these times.

Regardless of the slide errors, which are not a component of ICONN, ICONN is still able to track the sinusoidal fluctuations to within approximately $5 \%$ loss in visibility. The limiting factor is again attributable to a component external to ICONN and not inherent to it. Thus, it is concluded that in its current state, with little optimization performed, ICONN can track low frequency atmospheric-like disturbances to a level that results in a visibility loss of less than $5 \%$, and is well on its way to reaching the goal of less than a $1 \%$ visibility loss due to tracking errors.

\subsection{Moving forward}

With closed loop operation of ICONN demonstrated, the task becomes optimization. Little work was performed on detector characterization. Since closing the loop, we have been able to demonstrate frame rates of over 500 frames per second using the intended five line readout mode. ${ }^{2}$ Further detector characterization is ongoing. In addition, custom algorithm development is continuing that takes advantage of the ICONN modulators having the capability of performing $\lambda / 4$ steps that maximize the available visibility for the tracking algorithms. ${ }^{12}$

The cold optics have been delivered as well and are awaiting installment and alignment. The cold optics, coupled with the tilt/shear algorithms from the AAS, will minimize the alignment error currently seen by the detector. With these improvements, we expect ICONN to exceed its requirement of tracking visibilities to less than a $1 \%$ loss. 

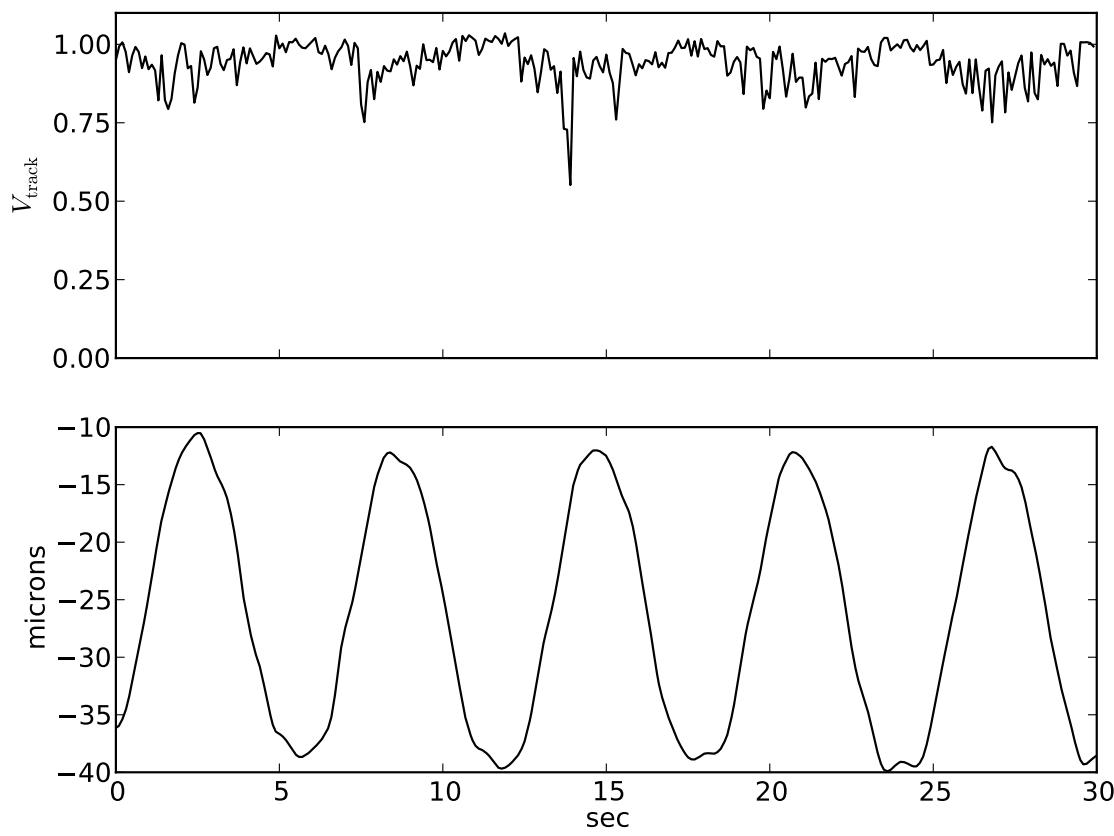

Figure 5: Plot of the mean tracking visibility and integrated offset. Note the periodicity of the tracking visibility.

\section{CONCLUSION}

We have demonstrated closed loop operation of the MROI fringe tracker ICONN. In a laboratory setup, ICONN was able to track a sinusoidal piston error such that no more than a $5 \%$ visibility loss was introduced. Careful characterization of the laboratory setup showed that a number of components, all external to ICONN, were the primary culprits limiting the experiment. Characterization and optimization of ICONN is ongoing but based on the results shown here, we are confident ICONN will be the work horse MROI requires.

\section{ACKNOWLEDGMENTS}

The Magdalena Ridge Observatory Interferometer is funded by the US Department of Transportation, the State of New Mexico, and New Mexico Tech with previous funding from the Navy Research Laboratory (NRL, agreement no. N00173-01-2-C902). MROI is hosted by New Mexico Tech at Socorro, NM, USA, in collaboration with the University of Cambridge (UK).

\section{REFERENCES}

1. Buscher, D. F., Creech-Eakman, M., Farris, A., Haniff, C. A., and Young, J. S., "The Conceptual Design of the Magdalena Ridge Observatory Interferometer," Journal Astronomical Instrumentation 2(02), 1340001 (2013).

2. Jurgenson, C. A., Santoro, F. G., McCracken, T. M., Young, J. S., Seneta, E. B., Buscher, D. F., CreechEakman, M. J., and Haniff, C. A., "ICONN - The Infrared Coherencing Nearest Neighbor Tracker," Journal Astronomical Instrumentation 02(02), 1340006 (2013).

3. Armstrong, J. T., Mozurkewich, D., Pauls, T. A., and Hajian, A. R., "Bootstrapping the NPOI: keeping long baselines in phase by tracking fringes on short baselines," in [Proc. SPIE Vol. 3350], 461-466 (1998).

4. Farris, A., Klinglesmith, D., Seamons, J., Torres, N., Buscher, D., and Young, J., "Software architecture of the Magdalena Ridge Observatory Interferometer," in [Proc. SPIE Vol. 7740], 77400R (2010). 
5. Shtromberg, A. V., Magdalena Ridge Observatory Interferometer automated alignment system, Master's thesis, New Mexico Institute of Mining and Technology (2013).

6. Shtromberg, A. V., Jurgenson, C. A., McCord, K. M., Olivares, A. M., Bloemhard, H. N., Santoro, F. G., Buscher, D. F., Haniff, C. A., Young, J. S., Torres, N. C., and Farris, A. R., "Magdalena Ridge Observatory Interferometer automated alignment system," in [Proc. SPIE Vol. 7734], (2010).

7. McCracken, T. M., Closing the loop on the MROI fringe tracker, PhD thesis, New Mexico Institute of Mining and Technology (2014).

8. McCracken, T. M., Jurgenson, C. A., Santoro, F., Shtromberg, A. V., Alvidrez, V., Torres, N., Dahl, C., Farris, A., Buscher, D. F., Haniff, C. A., Young, J. S., Seneta, E. B., and Creech-Eakman, M. J., "The MROI fringe tracker: closing the loop on ICoNN," in [Proc. SPIE Vol. 8445], 84451N (2012).

9. Traub, W. A., "Polarization effects in stellar interferometry," in [European Southern Observatory Conference and Workshop Proceedings], 29, 1029-1038 (1988).

10. Nyland, K., Jurgenson, C., Buscher, D. F., Haniff, C. A., Young, J. S., Lewis, J., and Schnell, R., "Custom beamsplitter and AR coatings for interferometry," in [Proc. SPIE Vol. 7734], (2010).

11. Basden, A. G. and Buscher, D. F., "Improvements for group delay fringe tracking," Monthly Notices of the Royal Astronomical Society 357(2), 656-668 (2005).

12. McCracken, T. M., Jurgenson, C. A., Haniff, C. A., Buscher, D. F., Young, J. S., and Creech-Eakman, M., "Open-loop phase shifting for fast acquisition of interferograms in low light levels.," Applied Optics 52(20), 4922-4932 (2013). 